\title{
Peculiarities of thermal expansion of quasi-two-dimensional organic conductor $\kappa-(\mathrm{BEDT}-\mathrm{TTF})_{2} \mathrm{Cu}\left[\mathrm{N}(\mathrm{CN})_{2}\right] \mathrm{Cl}$
}

\author{
A.V. Dolbin ${ }^{1}$, M.V. Khlistuck ${ }^{1}$, V.B. Eselson ${ }^{1}$, V.G. Gavrilko ${ }^{1}$, N.A. Vinnikov ${ }^{1}$, \\ R.M. Basnukaeva ${ }^{1}$, V.V. Danchuk ${ }^{1}$, V.A. Konstantinov ${ }^{1}$, and Y. Nakazawa ${ }^{2}$ \\ ${ }^{1}$ B. Verkin Institute for Low Temperature Physics and Engineering of the National Academy of Sciences of Ukraine \\ 47 Pr. Nauky, Kharkiv 61103, Ukraine \\ E-mail: dolbin@ilt.kharkov.ua \\ ${ }^{2}$ Department of Chemistry, Graduate School of Science, Osaka University, 1-1 Machikaneyama-cho, Toyonaka, \\ Osaka 560-0043, Japan
}

Received April 14, 2016, published online July 25, 2016

\begin{abstract}
Linear coefficient of thermal expansion $\alpha(T)$ of single crystal (BEDT-TTF) ${ }_{2} \mathrm{Cu}\left[\mathrm{N}(\mathrm{CN})_{2}\right] \mathrm{C} 1$ was studied along the crystal layers using the method of precise capacitive dilatometry in the temperature range $2-285 \mathrm{~K}$. It is positive in this direction over the entire temperature range. Anomalies of thermal expansion were observed at $29-30 \mathrm{~K}$ and $74-80 \mathrm{~K}$. The anomaly near $30 \mathrm{~K}$ is, apparently, due to the transition between the paramagnetic phase and the antiferromagnetic insulator state. Peak of $\alpha(T)$ at $78 \mathrm{~K}$ corresponds to a phase transition related to orientational disordering of ethylene groups in dimers of BEDT-TTF. A broad maximum of $\alpha(T)$ in the temperature range $40-70 \mathrm{~K}$, is apparently explained by fluctuations of charge within the dimers, and by spin fluctuations, which first increase with increasing temperature, and then decrease in process of thermal disordering of dimers.
\end{abstract}

PACS: 65.40. De Thermal expansion; thermomechanical effects; 63.22. Np Layered systems.

Keywords: organic molecular conductors, $(\mathrm{BEDT}-\mathrm{TTF})_{2} \mathrm{Cu}\left[\mathrm{N}(\mathrm{CN})_{2}\right] \mathrm{C} 1$, thermal expansion.

\section{Introduction. Quasi-two-dimensional organic conductors and their magnetic properties}

The problem of synthesis and study of the physical properties of low-dimensional organic molecular conductors and magnetic materials is at the junction area of solid state physics, materials science and organic synthesis. Organic (super) conductors and magnets on the basis of the cation - radical salts are unique materials with regard to their structure and properties. They are quasi-one-dimensional or quasi-two-dimensional systems, whose structure is characterized by the presence of conductive packages or layers of organic (metal organic) $\pi$-electron donors with the completely filled top electron band and strong electron correlations and interaction of charge carriers with the internal molecular vibrations. Due to this, such salts have unusual electronic properties distinguishing them from the elemental metals. The ground state in them can be metallic state, competing with the Mott insulator, the magnetically ordered or superconducting state, depending on the width and the filling of the initial metal band and the nature of the electron-phonon interaction. Organic conductive system attracted the constant attention of specialists that operate in the areas of basic research and applied topics, because these compounds are artificially synthesized metals and semiconductors. In general, organic molecules have a stand-alone structure with a closed electron shell, where two electrons with opposite spins take their HOMOs (highest occupied molecular orbitals). The charge carriers in such systems can be administered either by injecting electrons in lowest unoccupied molecular orbitals (LUMOs), or removal of electrons from HOMOs.

To date, there are at least three strategies for creating of conductive systems in organic solids [1]. The first is the introduction of mobile charge carriers by doping of cations or anions in the neutral crystal of $\pi$-conjugated molecules. For the first time such an organic semiconductor system was observed in pirilene doped with halogens [2]. Recent- 
ly, a new organic superconductor was created by doping solid picene with potassium [3]. Another well-known strategy of preparing of conductive systems is a synthesis of donor and acceptor salts [4]. Because the molecular structures and the directions of stacking of the molecules in these compounds have a strong anisotropy, the electronic band arising due to the overlap of the molecular orbitals of neighboring molecules is low-dimensional. The third strategy is the introduction of the charge carriers through the interface of the organic crystal and the insulating layer [5].

Thermodynamic studies of organic charge-transfer salts, obtained by a combination of donor and acceptor molecules, have been carried out for a number of compounds [6-8]. It is well known that the organic donor and acceptor charge transfer salts can have various types of electronic phases, such as superconducting spin density wave (SDW), charge density wave (CDW), antiferromagnetic (AFM) and so on. TTF-TCNQ salt is best known and has a long history of research since the first observation of metallic conductivity in 1973 [9]. In this material, TTF, TCNQ layers in the crystal form structures such as double columns. It is well known that the metal-insulator transition in these compounds occurs at $50 \mathrm{~K}$, due to the Peierls instability of the electronic structure of one-dimensional zones. In spite of numerous experiments, the details of the transition mechanism are not yet fully understood. In TSF-TCNQ salt, which is isostructural to TTF-TCNQ, the Peierls transition occurs in two stages at 28 and $32 \mathrm{~K}$, each of which is associated with the columns of TSF and TTF, respectively [10]. Electronic structure of the charge-transfer salts, consisting of BEDT-TTF (bisethlendithia tetrathiafuluvalene) and molecules of TCNQ was studied by investigating of the transport and magnetic properties. In this compound BEDT-TTF molecules tend to form a two-dimensional (2D) network structure separated with layers of TCNQ. These salts have several thermodynamically stable phases, usually referred to by the letters $\alpha, \beta, \theta, \lambda$ and $\kappa$, the packing pattern of the $\kappa$-phase differs distinctly from the others in the sense that it consists of two face-to-face BEDT-TTF molecules.

Donor and acceptor charge-transfer salts are convenient objects for the study of electronic and thermodynamic properties of quasi-one and two-dimensional systems. A number of salts has a simple composition $2: 1\left(\mathrm{D}_{2} \mathrm{X}\right.$ or $\left.\mathrm{A}_{2} \mathrm{Y}\right)$ and 1:1 (DX or AY), where the letters B and A denote the donor and acceptor molecules, and $\mathrm{X}$ and $\mathrm{Y}$ are anions and cations. Salts with a complex chemical composition, and also containing organic solvents such as $\mathrm{THF}, \mathrm{CH}_{2} \mathrm{Cl}_{2}$, $\mathrm{H}_{2} \mathrm{O}$ etc. are also sufficiently known. Among them 2:1 salts are the most studied, as they form a plurality of conductive and semi-conductive systems. [11-13]. In such compounds the above-mentioned various types of ground electronic states, such as SDW, CDW, an antiferromagnetic ordered by the charge (CO) and superconducting exist near the metal phase and are closely associated with each other. These salts have layered molecular structure and the conductivity occurs in the donor/acceptor layers. Such compounds include 2:1 salts consisting of several organic molecules: donors such as BEDT-TTF, and their counter ions.

It is known that charge transfer salts based on BEDT-TTF with $\kappa$-type structure have interesting physical properties due to the correlations in the $2 \mathrm{D}$ electron zones. In this structure a Mott insulators are implemented with antiferromagnetic transition in the region of $10-30 \mathrm{~K}$ as well as the transitions from a disordered magnetic state into a spin liquid, metallic and superconducting states with relatively high transition temperatures. Important parameters that determine the electronic properties of these compounds is the Coulomb interaction with the energy $U$, and the conduction band width $W$. $\kappa$-type structure is known as a highly dimerized structure in which donor dimers arranged almost orthogonal position and have a zigzag lattice structure (see Fig. 1).

$\kappa-(\mathrm{BEDT}-\mathrm{TTF})_{2} \mathrm{X}$ has a layered structure in which two-dimensional layers of dimers are arranged in a triangular anisotropic lattice. In particular, the organic salt $\kappa-(\mathrm{BEDT}-\mathrm{TTF})_{2} \mathrm{Cu}\left[\mathrm{N}(\mathrm{CN})_{2}\right] \mathrm{C} 1$ (thermal expansion of the single crystal was investigated in this paper), has a twolayer orthorhombic lattice, in which the layers of anions,

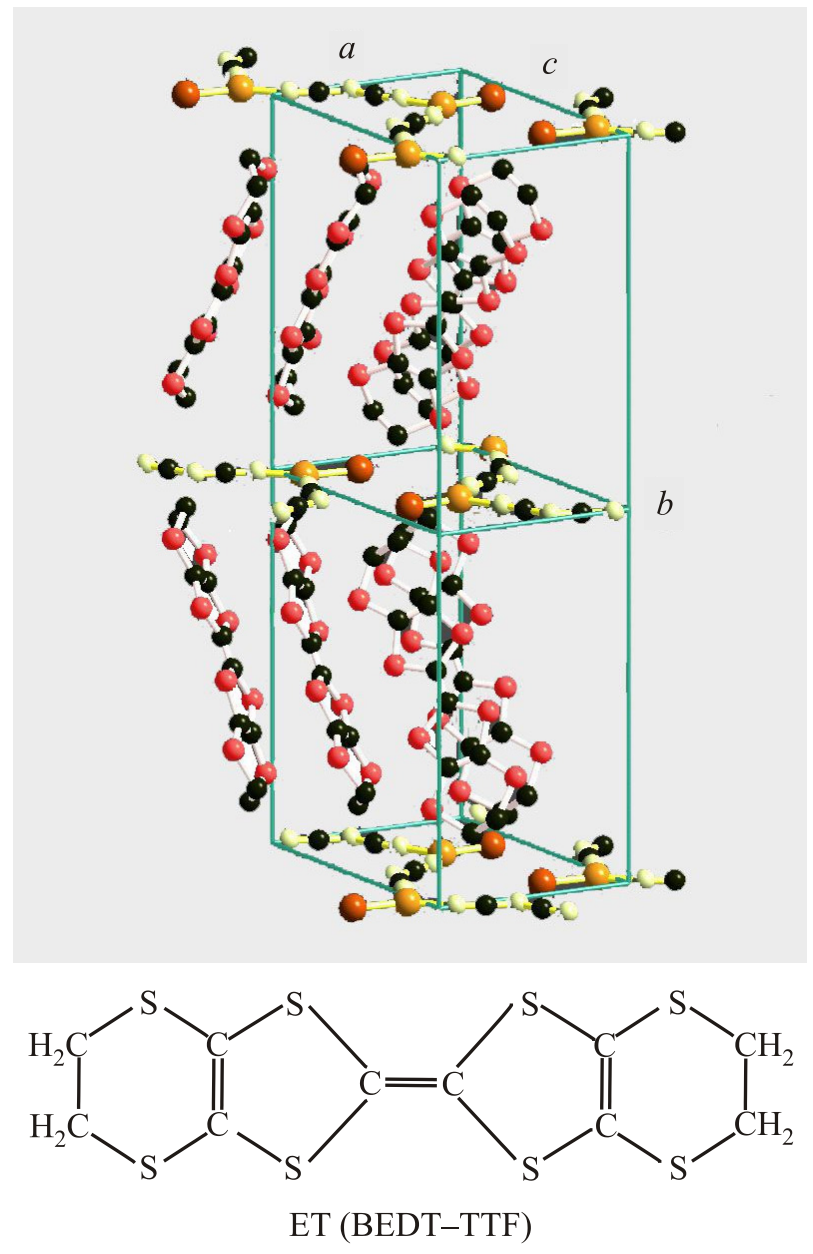

Fig. 1. Model of $\kappa-(\mathrm{BEDT}-\mathrm{TTF})_{2} \mathrm{X}$ structure, $\mathrm{X}$ are anions (insulating layers). 
which are located in the $a c$ plane, are perpendicular to the $b$-axis along which the flat BEDT-TTF dimers are situated (four per unit cell of the crystal lattice). The planes of dimers are inclined (along the $a$ axis) in the $a c$-plane and in opposite directions for adjacent layers. Conducting layers of cationic molecules BEDT-TTF ions are separated by insulating anion layers $\mathrm{Cu}\left[\mathrm{N}(\mathrm{CN})_{2}\right] \mathrm{C} 1$. BEDT-TTF molecules form a plane-parallel pairs (dimers), which are rotated by $90^{\circ}$ relative to the neighboring dimers (Fig. 1). Energy transfer within the dimer are generally significantly higher than other transferences, and therefore each dimer can be considered as a structural unit in the 2D plane. Rigid dimerization characteristic $\kappa$-type structure results in a unique situation in the electronic structure of these salts. Since each dimer is considered as a structural element in this ordering, the electronic state is interpreted as a simple effectively half-filled Mott-Hubbard state, and is determined by a subtle balance of band energy $W$, indoor/outdoor Coulomb energy of dimer ( $U$ and $V$, respectively), and the electron-phonon interaction between $\pi$-electrons existing in the low-dimensional lattice. If $U$ is greater than $W$, the system goes into Mott's insulating state (AFM ground state). On the other hand, in the region where $W$ exceeds $U$, the ground state is strongly correlated metallic state. In this area a relatively high temperature superconductivity $T_{c}$ $(\approx 10 \mathrm{~K})$ is observed [14-16].

It should also be noted that in organic charge transfer compounds wide variety of electronic and magnetic phases is observed at variation of the external parameters such as temperature $(T)$, pressure $(P)$, the magnetic field $(H)$, etc. This tendency increases sharply in the $\pi-d$ hybrid systems, where $\pi$-electrons in the organic layers have magnetic interaction with the $d$-electrons in the anion layers. The study of the relationship between superconductivity and longrange magnetic ordering is important for creating new organic conducting systems. A new class of multifunctional molecular materials gives an absolutely unique opportunity to watch the competition and co-operative relationship between two physical processes occurring in the crystal.

One major advantage of the use of molecular magnets is the wide range of possible chemical modification of the molecule. There are two major ways for this: changing the molecular structure of the donor and varying types of anions. Until recently, the role of anions in low-dimensional organic conductors was seen as secondary. Cationic and anionic layers are spatially well separated in the crystal, and the conduction band formed highest occupied molecular orbital of the radical cations. The anions performing the function of an electron acceptor, may affect the packaging donor molecules, which depends on the nature of the transport properties, but do not participate directly in the process of conduction. In the last two decades various kinds of molecular magnets have been synthesized and their chemical and physical properties have been studied in terms of microscopic and macroscopic positions using var- ious experimental methods. Been replaced real magnetic systems based on molecular magnets, composite magnetic systems come in recent years, which are cooperatively coupled with multiplet spin degrees of freedom. Such systems manifested in particular, the spin crossover and spinPeierls transition [17]. Recent examples of complex systems magnetoelectronic "spintronics" are known as "multiferroics" - materials having more than one magnetic order parameter at a time. In the case of complex systems with many degrees of freedom, the proper interpretation of the experimental results is often complex and ambiguous. The combination of structural and thermal research methods such as studies of thermal expansion is able greatly facilitate proper interpretation because the different processes of magnetic and structural ordering appear differently in the physical properties of these substances.

Thermal expansion measurements are the important tools for the study of fundamental properties of organic conductors. It is quite sensitive for volume change produced by phase transitions. Even for electronic transitions such as magnetic and superconductive ones, thermal expansion can probe them accurately. They makes possible to observe and identify the different types of phase transitions. Determination of the phase transition temperature provides information on the degree of ordering of various microscopic degrees of freedom of the molecules, atoms and the conduction electrons interacting with each other. Measurements of thermal expansion at low temperatures allow to observe the emergence of new phases and to obtain additional information on the nature of phase transitions. Furthermore, efficient use of thermodynamic measurements at low temperatures allows clarify the nature of the ground state and the low-energy excitation. Precision measurements of thermal expansion and heat capacity at low temperatures can also provide information about the specific phase transitions induced by quantum phenomena at low energies.

A major difficulty when measuring thermal expansion and other thermophysical properties of organic (super) conductors is cultivation of samples of sufficient mass. Crystals of organic charge-transfer compounds are grown usually by an electrochemical method or by diffusion from solutions of organic molecules. Typical weight of monocrystals is $1-100 \mathrm{mg}$, and their size is less than $1 \mathrm{~mm}$. Cultivation of large single crystals, suitable for measuring the thermal properties, is an extremely difficult task.

\section{The experimental technique}

Investigations of linear thermal expansion coefficient of the single crystal $\kappa-(\mathrm{BEDT}-\mathrm{TTF})_{2} \mathrm{Cu}\left[\mathrm{N}(\mathrm{CN})_{2}\right] \mathrm{C} 1$ was performed using a low-temperature capacitance dilatometer. Low-temperature part of the dilatometer consisting of a measuring cell and a meter of small displacements is shown in Fig. 2. 
Dilatometer was designed so that all elements, that may influence the measured values of the linear coefficient of thermal expansion of the sample were in the liquid helium bath at a constant temperature, and could not influence on the measurement results. To improve the accuracy of measurement the sensor of small displacements (3) (see Fig. 2(a)) and the high-frequency generator (4) are also at a temperature of liquid helium. The temperature of sample was measured by a silicon semiconductor thermometer (LakeShore DT-470). The required temperature of the sample is provided by a thermal switch (9) representing a capillary if necessary filled with liquid or gaseous helium, and a copper cold finger (10), having good thermal contact with the helium bath and equipped with a heater and a thermometer. Thermal insulation is ensured by maintaining of the sample in a vacuum of about $10^{-6}$ Torr. A simplified diagram of the dilatometer measuring cell is shown in the Fig. 2. Elements (1), (2), (5), (8) (see. Fig. 2(b)) were kept at a constant temperature of the helium bath. Thermometric block consisting of measuring thermometer, a thermometer to maintain the temperature, and the sample heater was mounted on the stage (4) of dilatometer and had a good thermal contact with it. The investigated sample (7) was placed at the same table. A temperature difference between top and bottom of the sample was controlled by a differential thermocouple (gold-iron alloy - copper), which measured a temperature difference between the dilatometer sample stage (4) and the thin strip of aluminum foil (3),

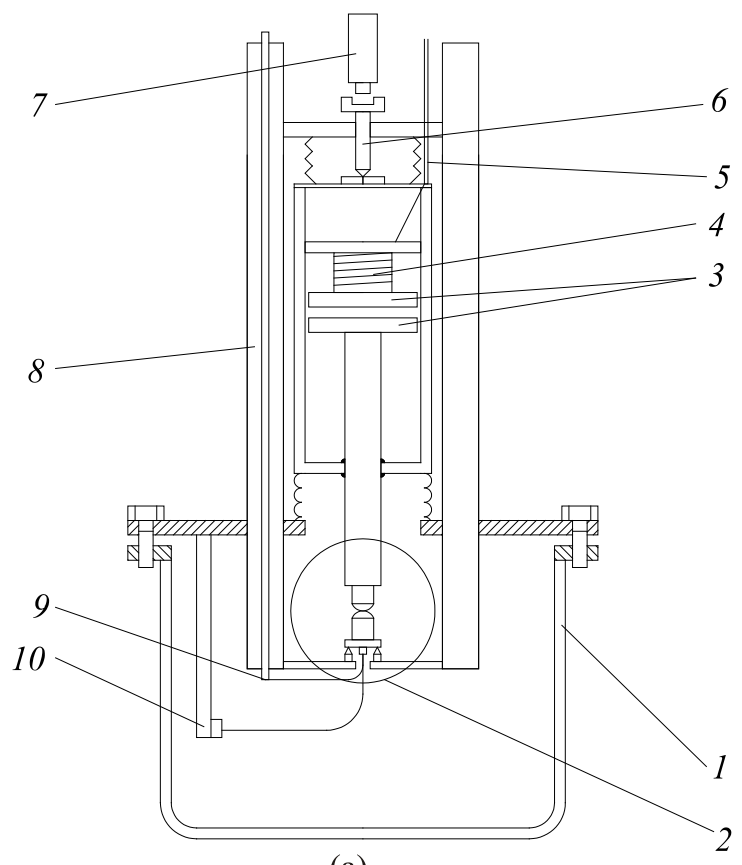

(a) which was located between the top of sample and the sapphire hemisphere (6), and had a thickness of $0.02 \mathrm{~mm}$ and a width of $7 \mathrm{~mm}$. Proper securing of a small sample of single crystal on the stage and sapphire lens centering relative to the sample were provided with a special Teflon mandrel (9). Thermal isolation the object table of dilatometer with structural units, as well as with sensor of small displacements was performed using point contacts sapphiresapphire. Such contacts are well established in dilatometers due to the high hardness and thermal conductivity of single crystal sapphire [18-19], providing the necessary rigidity and a large thermal point resistance. In view of this, on the one hand, the temperature gradient through the thickness of the sapphire plates can be considered negligible, on the other hand, all of the temperature difference between the sample and the fixing elements are concentrated in the point contacts sapphire-sapphire. As a result, the stock and the cell mount are during the experiment at a constant temperature of the helium bath and have no effect on the measured elongation. According to the data [18] thermal expansion coefficient of sapphire single crystal in the direction of about $60^{\circ}$ relative to its hexagonal axis is only $\alpha=1.2 T^{2.2} \cdot 10^{-11} \mathrm{~K}^{-1}$ at low temperatures. For the direction along the axis this value must be even smaller.

Therefore, in order to make the thermal expansion of the sapphire components minimal, they were cut from a single crystal of artificial sapphire so that the direction along which the measurements of the thermal expansion of

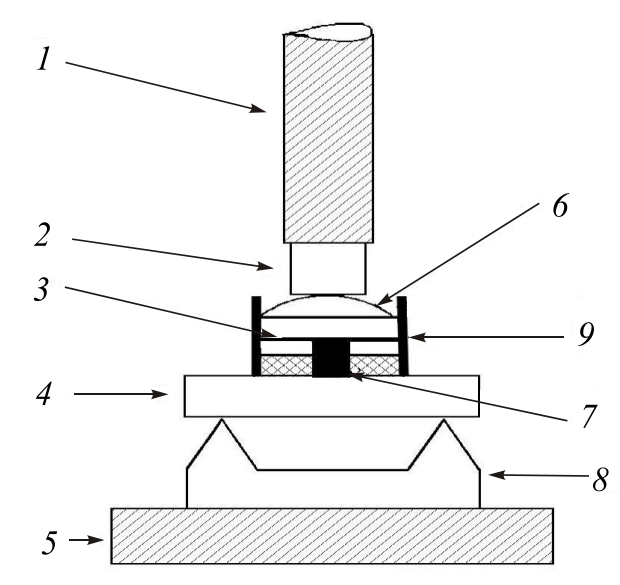

(b)

Fig. 2. Scheme of the low-temperature part of dilatometer, (a) vacuum cup (1); measuring cell (2); capacitive sensor of small displacements (3); high-frequency generator (4); coaxial feeder (5); micrometer screw (6); swivel rod (7); pumping line (8); thermal switch (9); cold finger with a block of heater and thermometer (10); (b) measuring cell: stock gauge of small displacements (1); sapphire stem tip (2); aluminum lining (3); sapphire sample stage of dilatometer (4); objective table (5); sapphire lens (6); investigated sample (7); sapphire stand of sample stage of dilatometer $(8)$; Teflon centering mandrel $(9)$. 
the samples were made coincides with hexagonal axis direction of the sapphire single crystals. To determine the true contribution of the sapphire elements and the aluminum foil to the thermal expansion, additional studies were conducted, which showed that this contribution is below the resolving power of the apparatus. Dilatometer element sensitive to small displacements was a two terminal capacitance sensor included in an oscillator circuit field effect transistor. Block composed of small displacements sensor and the generator was at a constant temperature bath of liquid helium. Stability of the generator was about $2 \cdot 10^{-8}$ within a few hours at an operating frequency of about $30 \mathrm{MHz}$, due to the high quality factor of the resonator at the temperature of liquid helium.

Calibration of the dilatometer was performed immediately before each series of measurements in two ways: i) by mechanically changing of the gap using a micrometer screw (Fig. 2(a), item (O)); ii) by measuring of the thermal expansion of a special sample of pure copper (copper purity was $99.999 \%$ ). When performing the calibration measurements no influence of thermal expansion of structural elements of dilatometer was observed in all temperature range 2-290 K. Maximum sensitivity of dilatometer was $2.6 \cdot 10^{-10} \mathrm{~cm} / \mathrm{Hz}$. High resolution of the dilatometer $\left(2 \cdot 10^{-9} \mathrm{~cm}\right)$ is due not only high stability of the generator, but also by specific measures to increase the thermal and vibration stability of the entire system as a whole. The change in length of the sample was defined both at increase and decrease in temperature. The pressure on the sample from the rod was about $1 \mathrm{~g} / \mathrm{cm}^{2}$ during the experiment. The coefficient of linear thermal expansion $\alpha$ was obtained as a derivative of the samples elongation $\Delta l / l$.

\section{Results and discussion}

The linear thermal expansion of the single crystal of $\kappa-(\mathrm{BEDT}-\mathrm{TTF})_{2} \mathrm{Cu}\left[\mathrm{N}(\mathrm{CN})_{2}\right] \mathrm{C} 1$ was studied in the temperature range of $2-285 \mathrm{~K}$. The single crystal was synthesized in Osaka University (Japan). The length of the sample in the measuring direction was $L=1.81 \mathrm{~mm}$, the width of the crystal in the direction perpendicular to the measurement $\sim 2 \mathrm{~mm}$. Figure 3 shows photographs of the studied sample, obtained with an optical microscope which step-like image clearly shows the layered structure of the crystal.
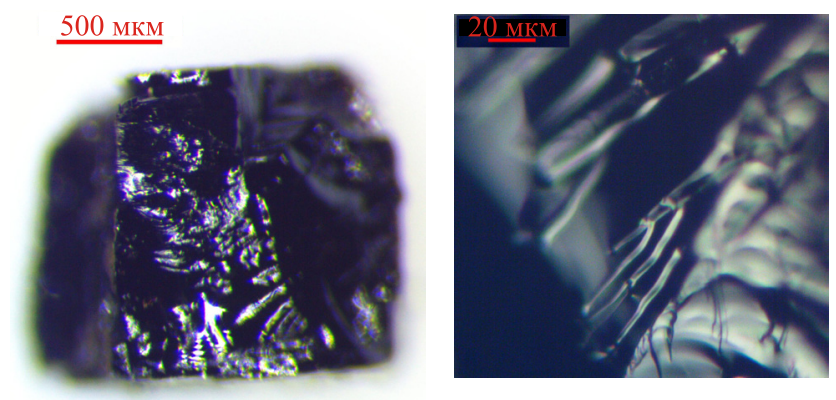

Fig. 3. Studied single crystals $\kappa-(\mathrm{BEDT}-\mathrm{TTF})_{2} \mathrm{Cu}\left[\mathrm{N}(\mathrm{CN})_{2}\right] \mathrm{C} 1$ under magnification.
Our experimental data on the relative elongation $\Delta l / l$ of the sample $\kappa-(\mathrm{BEDT}-\mathrm{TTF})_{2} \mathrm{Cu}\left[\mathrm{N}(\mathrm{CN})_{2}\right] \mathrm{C} 1$ were compared with the data of [6] (see Fig. 4). From their comparisons, one can conclude that obtained values of $\alpha$ correspond to the thermal expansion of the sample along the crystallographic $a$ axis ( $a$ is direction parallel $a s$-plane, i.e. along the crystal layers).

The measured value of linear thermal expansion was positive in the entire temperature range and is approximately $1.5 \cdot 10^{-7} \mathrm{~K}^{-1}$ at $2 \mathrm{~K}$. It increases with temperature, passes through the maximum at $60 \mathrm{~K}\left(4.5 \cdot 10^{-5} \mathrm{~K}^{-1}\right)$, shows then the minimum at $85 \mathrm{~K}\left(6 \cdot 10^{-6} \mathrm{~K}^{-1}\right)$ and increases again up to $6.5 \cdot 10^{-5} \mathrm{~K}^{-1}$ at $285 \mathrm{~K}$. There were anomalies of thermal expansion: a small at $T=29-30 \mathrm{~K}$ and clearly expressed one in the temperature range (74-80 K) (Fig. 5).

Figure 5 also shows the values of LCTE of single crystal $\kappa-(\mathrm{BEDT}-\mathrm{TTF})_{2} \mathrm{Cu}\left[\mathrm{N}(\mathrm{CN})_{2}\right] \mathrm{Cl}$ along the $\mathrm{c}$ axis according to [20]. It is noticeable that the temperature ranges in which anomalies of thermal expansion along the $a$ and $c$ axes manifest themselves substantially coincide in both cases. The anomaly near $30 \mathrm{~K}$, is apparently due to the transition from the paramagnetic (high-conducting) phase (stable above $30 \mathrm{~K}$ ) to the state of the antiferromagnetic insulator (AFI) [21], which in framework of the two-dimensional Hubbard model (2D Hubbard model) is described as a Mott insulator [22]. This transition, as has been said in the first section, caused by the competition of local energy of dimers electron states $U$ and the width of the conduction band $W$.

Broad maximum $\alpha(T)$ in the temperature range 40-70 K, probably, may be linked with intra-dimer charge and spin fluctuations [23], that firstly increases with increasing temperature, and decreases then on account of the thermal disordering in the crystal. Peak $\alpha(T)$ at a temperature of about $78 \mathrm{~K}$ corresponds to a phase transition due to the process of disinhibition of fluctuations of ethylene groups at the ends of the molecules BEDT-TTF, resulting in ori-

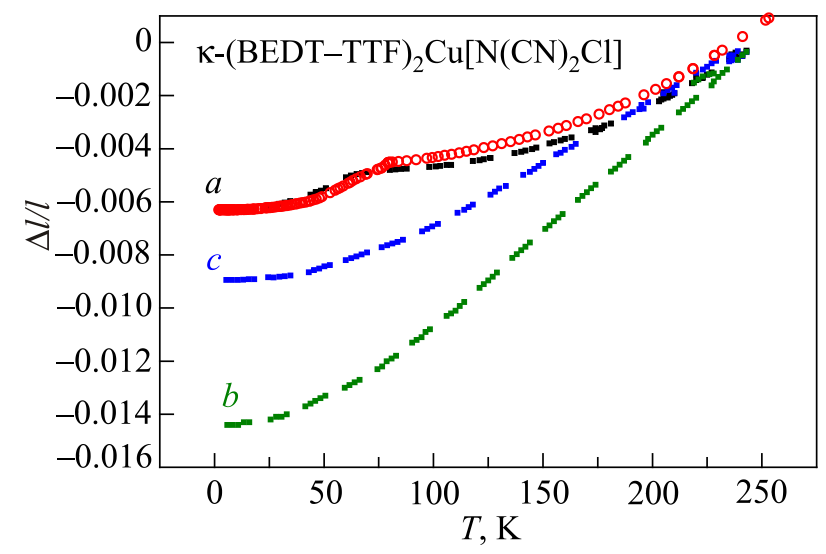

Fig. 4. Relative elongation $\Delta l / l$ for $\kappa-(\mathrm{BEDT}-\mathrm{TTF})_{2} \mathrm{Cu}\left[\mathrm{N}(\mathrm{CN})_{2}\right] \mathrm{C} 1$ sample, open circles is this work, dashed lines correspond to data of [6] (along the three principal axes $(a, b, c)$ ). 

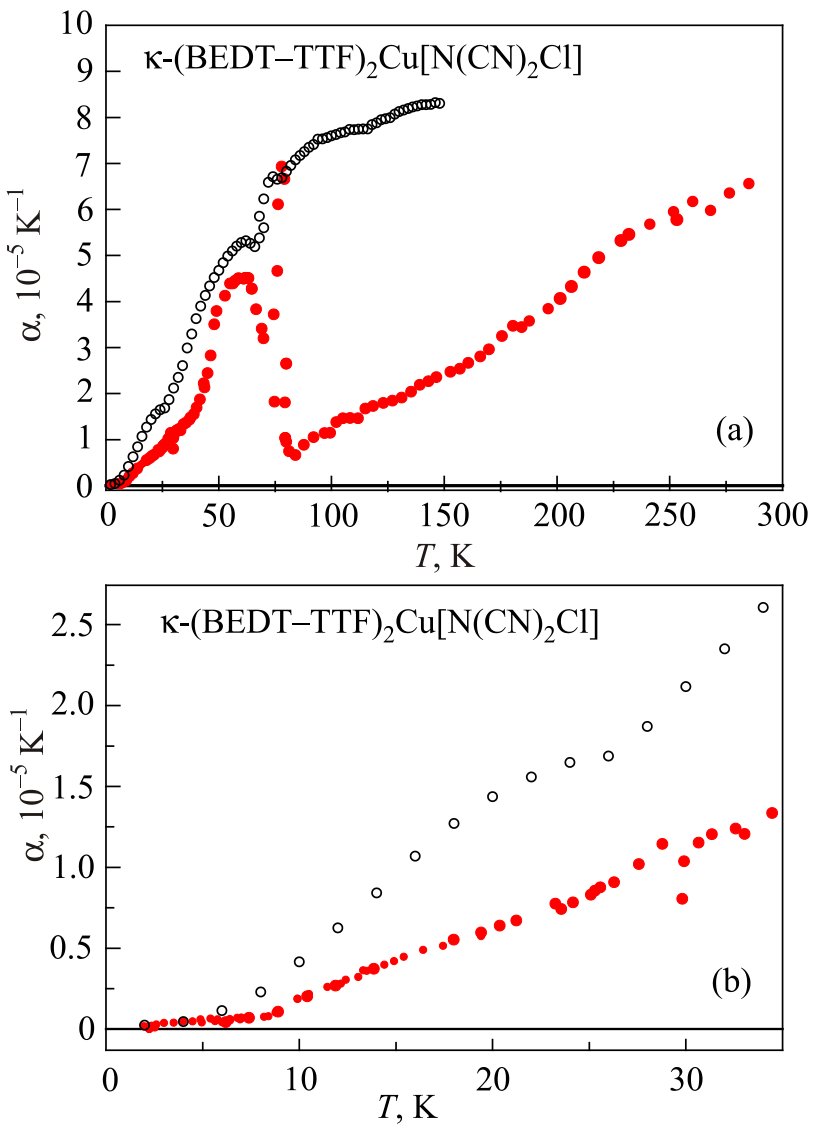

Fig. 5. The coefficients of linear thermal expansion of $\kappa$-(BEDT$\mathrm{TTF})_{2} \mathrm{Cu}\left[\mathrm{N}(\mathrm{CN})_{2}\right] \mathrm{C} 1$ in the direction of the crystallographic axis (a) (filled circles, this work) and along the $(c)$ axis (open circles) according to [20].

entation ordering of dimers disturbed with increasing in temperature [24-26].

\section{Conclusions}

The thermal expansion of the single crystal of $\kappa-(\mathrm{BEDT}-\mathrm{TTF})_{2} \mathrm{Cu}\left[\mathrm{N}(\mathrm{CN})_{2}\right] \mathrm{C} 1$ was investigated in the temperature range of $2-285 \mathrm{~K}$ along the crystallographic axis $(a)$ (i.e., along the crystal layers). The measured thermal expansion coefficient $\alpha(T)$ of the sample in this direction is positive in the entire temperature range. It was detected abnormalities of thermal expansion in the temperature ranges $29-30 \mathrm{~K}$ and $74-80 \mathrm{~K}$. The anomaly near $30 \mathrm{~K}$ is apparently explained by the transition of the sample from the paramagnetic phase in the antiferromagnetic insulator state. Peak $\alpha(T)$ at a temperature of about $78 \mathrm{~K}$ corresponds to a phase transition due to the orientational disordering in ethylene groups of dimers BEDT-TTF. Broad maximum $\alpha(T)$ in the temperature range $40-70 \mathrm{~K}$, probably, may be linked with intra-dimer charge and spin fluctuations that firstly increase with increasing temperature, and decreases then on account of the thermal disordering in the crystal.

The authors thank Prof. A.I. Prokhvatilov for the fruitful discussion.
1. M. Sorai, Y. Nakazawa, M. Nakano, and Y. Miyazaki, Chem. Rev. 113, PR 41 (2006).

2. H. Akamatsu, H. Inokuchi, and Y. Matsunaga, Nature 173, 168 (1954).

3. R. Mitsuhashi, Y. Suzuki, Y. Yamanari, H. Mitamura, T. Kambe, N. Ikeda, H. Okamoto, A. Fujiwara, M. Yamaji, N. Kawasaki, Y. Maniwa and Y. Kubozono, Nature, 464, 76 (2010).

4. T. Ishiguro, K. Yamaji, and G. Saito, Organic Superconductors, Berlin, NY: Springer (1996).

5. H. Klank, Organic Electronics Materials, Manufacturing and Application, Weinheim Germany: Wilet-VCH Verlag; Bao. Z. Locklin, J. Organic Field-Effect Transistors, Optical Science and Engineering Series, CRC Press, London (2006).

6. M. Kund, H. Müller, N.D. Kushch, K. Andres, and G. Saito, Synthetic Metals 70, 951(1995).

7. A. Naito, Y. Nakazawa, K. Saito, H. Taniguchi, K. Kanoda, and M. Sorai, Phys. Rev. B 71, 054514 (2005).

8. T. Ishikawa, Y. Nakazawa, S. Yamashita, M. Oguni, K. Saito, K. Takimiya, and T. Otsubo, J. Phys. Soc. Jpn. 75, 074606 (2006).

9. L.B. Coleman, J.J. Cohen, D.J. Sandman, F.G. Yamagishi, A.F. Garito, and A.J. Heeger, Solid State Commun. 12, 1125 (1973).

10. K. Saito, Y. Yamamura, H. Akutsu, M. Takeda, H. Asaoka, H. Nishikawa, I. Ikemoto, and M. Sorai, J. Phys. Soc. Jpn. 68, 1277 (1999).

11. H. Seo, C. Hotta, and H. Fukuyama, Chem. Rev. 104, 5005 (2004).

12. H. Mori, J. Phys. Soc. Jpn. 75, 051003 (2006).

13. V.A. Bondarenko, S. Uji, T. Terashima, C. Terakura, S. Tanaka, S. Maki, J. Yamada, and S. Nakatsuji, Synth. Met. 120, 1039 (2001).

14. K. Kanoda, Physica C 287, 299 (1997).

15. K. Miyagawa, K. Kanoda, and A. Kawamoto, Chem. Rev. 104, 5635 (2004)

16. K. Kanoda, J. Phys. Soc. Jpn. 75, 051007 (2006).

17. K. Fukuroi, K. Takahashi, T. Mochida, T. Sakurai, H. Ohta, T. Yamamoto, Y. Einaga, and H. Mori, Angew. Chem. Int. Ed. Engl. 53, 1983 (2014).

18. R.H. Carr and C.A. Swenson, Cryogenics 4, 76 (1964).

19. K.O. McLean, C.A. Swenson, and C.R. Case, J. Low Temp. Phys. 7, 77 (1972).

20. J. Müller, M. Lang, F. Steglich, J. A. Schlueter, A. M. Kini, and T. Sasaki, Phys. Rev. B 65, 144521 (2002).

21. U. Welp, S. Fleshler, W.K. Kwok, G.W. Crabtree, K.D. Carlson, H.H. Wang, U. Geiser, J.M. Williams, and V.M. Hitsman, Phys. Rev. Lett. 69, 840 (1992)

22. H. Kino and H. Fukuyama, J. Phys. Soc. Jpn. 65, 2158 (1996).

23. A. Painelli, A. Girlando, and A. Fortunelli, Phys. Rev. B 64, 054509 (2001).

24. K. Kanoda, Hyperfine Int. 104, 235 (1997). 